PROCEEDINGS OF THE

AMERICAN MATHEMATICAL SOCIETY

Volume 126, Number 7, July 1998, Pages 2077-2081

S 0002-9939(98)04315-9

\title{
THE VALENCE OF HARMONIC POLYNOMIALS
}

\author{
A. S. WILMSHURST
}

(Communicated by Albert Baernstein II)

\begin{abstract}
The paper gives an upper bound for the valence of harmonic polynomials. An example is given to show that this bound is sharp.
\end{abstract}

Interest in harmonic mappings in the complex plane has increased due to the publication of [1] in 1984. Most of this interest has centered on univalent harmonic mappings. It is, however, the purpose of this paper to present the fundamental valence results of complex valued polynomials which are harmonic in the plane. Specifically a harmonic polynomial is a function $P(z)=\overline{S(z)}+T(z)$ where $S(z)$ and $T(z)$ are analytic polynomials. The degree of $P$ is defined as the larger of the degrees of $S$ and $T$. Lyzzaik studied the local topology of harmonic mappings in [4]; in particular he put a bound on the local valence of $P$ but did not obtain a global valence bound. In this paper it will be shown that, apart from certain degenerate examples, $P(z)$ is at most $n^{2}$-valent, where $n$ is the degree of $P$. An example is exhibited to show this to be the sharp bound.

To prove the $n^{2}$ valence bound we need the following classical result from algebraic geometry; a proof may be found in [2].

Theorem 1 (Bézout's Theorem). Let $A$ and $B$ be relatively prime polynomials in the real variables $x$ and $y$ with real coefficients, and let $\operatorname{deg} A=n$ and $\operatorname{deg} B=m$. Then the two algebraic curves $A(x, y)=0$ and $B(x, y)=0$ have at most $m n$ points in common.

To apply Bézout's theorem to the valence problem we restate it in an equivalent form.

Theorem 2 (Alternate form of Bézout's Theorem). Let $A$ and $B$ be polynomials in the real variables $x$ and $y$ with real coefficients. If $\operatorname{deg} A=n$ and $\operatorname{deg} B=m$, then either $A$ and $B$ have at most mn common zeros or have infinitely many common zeros.

Proof. If $A$ and $B$ are relatively prime, then, by Bézout's theorem, the polynomial equations $A(x, y)=0$ and $B(x, y)=0$ have at most $m n$ common zeros. Otherwise $A$ and $B$ are not relatively prime and they have a greatest common factor, say $C$. Let $\operatorname{deg} C=q$. If $C(x, y)=0$ has no solutions, then $A$ and $B$ have at most $(n-q)(m-q)$ common zeros. But if there exists a point on $C(x, y)=0$ with either $\frac{\partial C}{\partial x} \neq 0$ or $\frac{\partial C}{\partial y} \neq 0$, then, by the implicit function theorem, $C(x, y)=0$

Received by the editors June 26, 1995 and, in revised form, September 20, 1996 and January 2, 1997.

1991 Mathematics Subject Classification. Primary 30C55.

(C) 1998 American Mathematical Society 
is an arc in some neighbourhood of the point, and in this case $A$ and $B$ have infinitely many common zeros. The only other possibility left to consider is that of $\frac{\partial C}{\partial x}=\frac{\partial C}{\partial y}=0$ at every point on $C(x, y)=0$. Clearly if a factor of $C$ is repeated it will give the same zeros, and since we are only concerned with the number of distinct zeros without regard for any multiplicity, we may assume that $C(x, y)=0$ has no repeated factors. Therefore $C$ and $\frac{\partial C}{\partial y}$ do not have any common factors involving $y$. But by a preliminary rotation, we may assume that there are no factors of $C$ which contain only $x$. Therefore we may now assume $C$ and $\frac{\partial C}{\partial y}$ are relatively prime. Thus, by Bézout's theorem, $C$ and $\frac{\partial C}{\partial y}$ have at most $q(q-1)$ common zeros. It follows that $C$ has at most $q(q-1)$ zeros, since every zero of $C$ is a zero of $\frac{\partial C}{\partial y}$. Applying Bézout's theorem again, we see at once that $A$ and $B$ have at most $(n-q)(m-q)+q(q-1) \leq n m-q$ common zeros, since $2 q \leq n+m$.

We may write $P(z)$ in terms of its real and imaginary parts, so $P(x+i y)=$ $A(x, y)+i B(x, y)$. Clearly Theorem 2 gives immediately the result that $P(z)$ either has at most $n^{2}$ zeros, or has infinitely many zeros. So in order to prove $n^{2}$ valence it is now sufficient to determine conditions on $P(z)$ which imply finite valence. To do this we will require the following, previously unpublished, theorem of T. SheilSmall.

Theorem 3. Let a function $f$ be harmonic in some domain $D$ and have a sequence of distinct zeros $\left(z_{m}\right)_{m=0}^{\infty}$ converging to a point $z^{*}$ in $D$. Then $f(z) \equiv 0$ on some simple analytic arc containing $z^{*}$ as an interior point. Further, there are at most finitely many such arcs unless $f(z) \equiv 0$ in $D$.

Proof. Without loss of generality it may be assumed that $z^{*}=0$. A function $f$ is harmonic in a disc if, and only if, it can be expressed as the sum of an analytic function and the conjugate of an analytic function. So in some neighbourhood of the origin $f$ may be written as

$$
f(z)=\sum_{n=1}^{\infty} a_{n} z^{n}+\sum_{n=1}^{\infty} a_{-n} \bar{z}^{n} .
$$

If $a_{n}=0$ for every $n$, then $f(z) \equiv 0$ in the neighbourhood and so vanishes identically in the domain. Otherwise let $k$ be the lowest positive integer such that $a_{k} \neq 0$ or $a_{-k} \neq 0$. Then

$$
\lim _{m \rightarrow \infty} \frac{f\left(z_{m}\right)}{\left|z_{m}\right|^{k}}=0
$$

or

$$
\lim _{m \rightarrow \infty} a_{k} e^{i k \theta_{m}}+a_{-k} e^{-i k \theta_{m}}=0
$$

where $z_{m}=\left|z_{m}\right| e^{i \theta_{m}}$. This is possible only if $\left|a_{k}\right|=\left|a_{-k}\right|$, so $f$ has the form $f(z)=\overline{z^{k} g(z)}+z^{k} h(z)$ where $g$ and $h$ are analytic functions with $|g(0)|=|h(0)| \neq 0$. The derivatives of $\left(z^{k} h(z)\right)^{1 / k}$ and $\left(z^{k} g(z)\right)^{1 / k}$ are non-zero at 0 so the functions are univalent in some neighbourhood of 0 . Therefore $z^{k} g(z)=p(z)^{k}$ and $z^{k} h(z)=$ $-(q(z))^{k}$ where $p$ and $q$ are analytic and univalent in a neighbourhood of 0 . Now for $m$ large enough $q\left(z_{m}\right)^{k}=\overline{p\left(z_{m}\right)^{k}}$, and so for infinitely many $m$ it is true that

$$
\overline{p\left(z_{m}\right)}=\omega q\left(z_{m}\right) \stackrel{\text { def }}{=} r\left(z_{m}\right)
$$


where $\omega$ is some fixed $k^{\text {th }}$ root of unity. But $p$ has an inverse, so writing $w_{m}=p\left(z_{m}\right)$ we have $z_{m}=p^{-1}\left(w_{m}\right)$ and (1) becomes

$$
\bar{w}_{m}=r\left(p^{-1}\left(w_{m}\right)\right) \stackrel{\text { def }}{=} s\left(w_{m}\right) .
$$

Multiplying (2) by $e^{-i \theta / 2}$ where $s^{\prime}(0)=e^{i \theta}$ and defining $\zeta=e^{i \theta / 2} w$ and $t(\zeta)=$ $e^{-i \theta / 2} s\left(e^{-i \theta / 2} \zeta\right)$ we obtain $\bar{\zeta}_{m}=t\left(\zeta_{m}\right)$. Transforming again, this time to the $\sigma$ plane gives

$$
\sigma=u(\zeta)=\frac{1}{2}(\zeta+t(\zeta)), \quad u^{\prime}(0)=1
$$

But $u$ is univalent in some neighbourhood of 0 , so has an inverse there. Therefore by defining $\sigma_{m}=u\left(\zeta_{m}\right)$ we have

$$
\sigma_{m}=\frac{1}{2}\left(\zeta_{m}+t\left(\zeta_{m}\right)\right)=\operatorname{Re}\left(\zeta_{m}\right)=\operatorname{Re}\left(u^{-1}\left(\sigma_{m}\right)\right)
$$

Therefore $\operatorname{Re}\left(u^{-1}\left(\sigma_{m}\right)-\sigma_{m}\right)=0$. What we have now is a real harmonic function $v(\sigma)=\operatorname{Re}\left(u^{-1}(\sigma)-\sigma\right)$ that is zero at every $\sigma_{m}$ and further (since $u$ is univalent and the $\zeta_{m}$ are distinct) the $\sigma_{m}$ are distinct real numbers which tend to zero. Now for $\sigma$ real $v(\sigma)$ is an analytic function of $\sigma$, so $v(\sigma) \equiv 0$ for all real $\sigma$ in some neighbourhood of the origin. Performing all of the above transformations in reverse we see that $f(z)=0$ for all $z$ on the curve $p^{-1}\left(e^{-i \theta / 2} u^{-1}(\sigma)\right)$, where $\sigma$ is real and sufficiently small. Thus $f(z) \equiv 0$ on an open arc through 0 , the arc having an injective analytic parametrization. The other $k^{\text {th }}$ roots of unity may give $k-1$ more such open arcs.

Theorem 4. Let $f$ be a function harmonic in the (entire) complex plane. If $\liminf _{z \rightarrow \infty}|f(z)|>0$, then $f$ has finitely many zeros.

Proof. By hypothesis $f(z) \neq 0$ outside some disc. If $f$ does have infinitely many zeros, then there exists a sequence of (distinct) zeros of $f$ converging to a point in the disc. By Theorem $3 f(z) \equiv 0$ on an analytic simple arc through this point. This arc may then be continued through its end points, and so on for each subsequent continuation. Since the zero set of $f$ is bounded this continuation process must eventually result in the formation of a loop [6]. But $f(z) \equiv 0$ on this loop and so, by the maximum principle, $f(z) \equiv 0$ in the entire plane, a contradiction. Thus $f(z)$ has only finitely many zeros.

Combining Theorems 2 and 4 we now have

Theorem 5. Let $P(z)$ be an harmonic polynomial of degree $n$. If $\lim _{z \rightarrow \infty} P(z)=$ $\infty$, then $P(z)$ has at most $n^{2}$ zeros.

Although $\lim _{z \rightarrow \infty} P(z)=\infty$ implies an upper bound on the number of zeros of $P$ it is too general to imply the existence of any zeros. For example take $P(z)=$ $\bar{z}^{2}+\bar{z}+1-z+z^{2}$. Then $\operatorname{Re}(P(x+i y))=2 x^{2}-2 y^{2}+1$ and $\operatorname{Im}(P(x+i y))=-2 y$ so $P(z) \rightarrow \infty$ as $z \rightarrow \infty$. But when $\operatorname{Im}(P(z))=0$ it is clear that $\operatorname{Re}(P(z)) \geq 1$, so $P$ cannot have any zeros.

To provide an example of a polynomial of degree $n$ with as many as $n^{2}$ zeros we begin with the observation that the level curve $\operatorname{Im}\left(z^{n}\right)=0$ is composed of $n$ straight lines passing through the origin. Denote by $\mathcal{F}_{1}$ the family of lines

$$
\operatorname{Im}\left(e^{-i \pi / 4} z^{n}\right)=0
$$


obtained by rotating the above $n$ lines by an angle of $\pi / 4 n$. Denote by $\mathcal{F}_{2}$ the family of lines

$$
\operatorname{Im}\left(e^{i \pi / 4}(z-1)^{n}\right)=0
$$

obtained by rotating the original lines by $-\pi / 4 n$ and translating by 1 to the right. Looking at the two families of lines, we see that no line in $\mathcal{F}_{1}$ is parallel to a line in $\mathcal{F}_{2}$. Thus each line in $\mathcal{F}_{1}$ intersects every line of $\mathcal{F}_{2}$ exactly once. So the two level curves $\operatorname{Im}\left(e^{-i \pi / 4} z^{n}\right)=0$ and $\operatorname{Im}\left(e^{i \pi / 4}(z-1)^{n}\right)=0$ intersect in exactly $n^{2}$ points. That all these points are distinct follows easily since each line in $\mathcal{F}_{1}$ intersects $\mathcal{F}_{2}$ in exactly $n$ distinct points, none of which is the origin, and the lines of $\mathcal{F}_{1}$ only intersect each other at the origin.

Therefore the harmonic polynomial

$$
\operatorname{Im}\left(e^{-i \pi / 4} z^{n}\right)+i \operatorname{Im}\left(e^{i \pi / 4}(z-1)^{n}\right)
$$

of degree $n$ has exactly $n^{2}$ distinct zeros. We can rewrite this polynomial in the form

$$
\frac{1}{2 i}\left(e^{-i \pi / 4} z^{n}-e^{i \pi / 4} \bar{z}^{n}\right)+\frac{1}{2}\left(e^{i \pi / 4}(z-1)^{n}-e^{-i \pi / 4}(\bar{z}-1)^{n}\right) .
$$

Multiplying by $-2 e^{i \pi / 4}$ and swapping $z$ and $\bar{z}$ gives the more pleasing polynomial

$$
Q(z)=z^{n}+(z-1)^{n}+i \bar{z}^{n}-i(\bar{z}-1)^{n} .
$$

In fact

$$
\left(\frac{1}{i}\right)^{n} Q(i z+1 / 2)=(z-i / 2)^{n}+(z+i / 2)^{n}+i(-\bar{z}-i / 2)^{n}-i(-\bar{z}+i / 2)^{n}
$$

is a polynomial with $n^{2}$ zeros and all its coefficients real.

Remark 1. Sheil-Small defined the following class in [5]. Let $\Gamma$ be a convex Jordan curve parametrized by the homeomorphism $F\left(e^{i t}\right):[0,2 \pi] \rightarrow \Gamma$. Define $f\left(e^{i t}\right)=$ $F\left(e^{i \phi(t)}\right)$ where $\phi$ is an increasing function satisfying $\phi(2 \pi)-\phi(0) \leq 2 \pi N$. We can extend $f$ into the unit disc by Poisson's formula, the class so defined is written $\widetilde{\Phi}(\Gamma, N)$. It is still an open problem to determine an upper bound on the valence of non-degenerate members of $\widetilde{\Phi}(\Gamma, N)$, but $Q(z)$ combined with Lyzzaik's method of [3] shows that the upper bound for the valence (if it exists) of such functions is at least $N^{2}$.

Remark 2. Let $P(z)=\overline{S(z)}+T(z)$, where $\operatorname{deg}(z)=m$ and $\operatorname{deg} T(z)=n$, satisfy $P(z) \rightarrow \infty$ as $z \rightarrow \infty$. By conjugating $P(z)$, if necessary, we may assume that $n \geq m$. For $m=n$ and $m=n-1$ we have shown the valence bound $n^{2}$ to be sharp, but for $m<n-1$ it would be surprising if it were still possible to obtain $n^{2}$ valence. I conjecture the correct bound to be $m(m-1)+3 n-2$ for $1 \leq m \leq n-1$. For $m=1$ this becomes $\bar{z}+T(z)$ is at most $3 n-2$ valent, or equivalently, that the number of fixed points of the conjugate of an analytic polynomial of degree $n$ is at most $3 n-2$.

Remark 3. For $P(z)=a_{-n} \bar{z}^{n}+\cdots+a_{-1} \bar{z}+a_{0}+a_{1} z+\cdots+a_{n} z^{n}$ with $\left|a_{-n}\right| \neq\left|a_{n}\right|$ we can use the affine transformation $P(z) \mapsto \overline{P(z)}-\left(a_{n} / a_{-n}\right) P(z)$ to reduce the coefficient of $\bar{z}^{n}$ to zero. From this it can be deduced that the resultant of $P(z)$ 
and $\overline{P(z)}$ with respect to $\bar{z}$ is a polynomial in $z$ of degree $n^{2}$. It can then be shown that

(i) $\operatorname{Re}(P(x, y))$ and $\operatorname{Im}(P(x, y))$ are relatively prime. So, by Bézout's theorem, we have a new proof of Theorem 5 in the case $\left|a_{-n}\right| \neq\left|a_{n}\right|$.

(ii) $\sum_{j}\left|m\left(P, z_{j}\right)\right| \geq n$.

(iii) $\sum_{j}\left|m\left(P, z_{j}\right)\right|^{2} \leq n^{2}$.

Here $m\left(P, z_{j}\right)$ is the topological multiplicity of the zero $z_{j}$. Further details can be found in $[6]$.

\section{REFERENCES}

[1] J. Clunie and T. Sheil-Small, Harmonic univalent functions, Ann. Acad. Sci. Fenn. Ser. A I Math. 9 (1984), 3-25. MR 85i:30014

[2] F. Kirwan, Complex Algebraic Curves, Cambridge University Press (1992). MR 93j:14025

[3] A. Lyzzaik, On the valence of some classes of harmonic maps, Math. Proc. Cambridge Philos. Soc. 110 (1991), 313-325. MR 92f:31001

[4] _ Local properties of light harmonic mappings, Canad. J. Math. 44 (1992), 135-153. MR 93e: 30048

[5] T. Sheil-Small, On the Fourier series of a finitely described convex curve and a conjecture of H. S. Shapiro, Math. Proc. Cambridge Philos. Soc. 98 (1985), 513-525. MR 86m:42009

[6] A. S. Wilmshurst, Complex harmonic mappings and the valence of harmonic polynomials, D. Phil. thesis, University of York, England, 1994.

Department of Mathematics, University of York, York Y01 5DD, England 